\title{
Psicooncología
}

ISSN: $1696-7240$

https://dx.doi.org/10.5209/psic. 77753

\section{Síndrome de sobrecarga y estrategias de afrontamiento en cuidadores principales de pacientes oncológicos pediátricos}

\author{
Paola Daniela Reyna-García ${ }^{1}$; Tomás Caycho-Rodríguez ${ }^{2}$; Claudio Rojas-Jara ${ }^{3}$
}

Recibido: 25 de marzo de 2021 / Aceptado 1 de junio de 2021

Resumen: Objetivo: Los cuidadores oncológicos pediátricos se encontrarán con circunstancias que llegarán a desafiar sus recursos personales para afrontar las diversas demandas propias del cuidado. Por lo tanto, el objetivo del estudio fue determinar la relación entre el síndrome de sobrecarga y las estrategias de afrontamiento en cuidadores de pacientes oncológicos pediátricos. Método: Participaron 93 cuidadores principales de pacientes oncológicos pediátricos $(69,9 \%$ mujeres, $30,1 \%$ varones, edad promedio $=37,20$ años, $\mathrm{DE}=11,32$ ), seleccionados de forma no probabilística. Se aplicó la Escala de Sobrecarga del Cuidador y el Cuestionario COPE-28. Se utilizaron estadísticos correlacionales y comparativos para el análisis de datos. Resultados: Los resultados indican la presencia de correlaciones significativas e inversas que varían entre $r=-, 25$ y $r=-, 56$. Sin embargo, no se evidenció una asociación significativa entre las dimensiones de la sobrecarga del cuidador y el uso de sustancias. Por otro lado, aquellos cuidadores que utilizan estrategias poco adaptativas, son más propensos a generar sobrecarga frente a su rol, influyendo en su vida personal, familiar y social. Conclusión: Existe una relación significativa entre el síndrome de sobrecarga y las estrategias de afrontamiento. Además, se identifican diferencias significativas en la sobrecarga según el lugar de procedencia y las horas dedicadas al cuidado.

Palabras clave: Síndrome de sobrecarga, estrategias de afrontamiento, cuidadores principales, pacientes oncológicos pediátricos.

\section{[en] Burden syndrome and coping strategies in caregivers of pediatric cancer patients}

\footnotetext{
Abstract: Objective: Pediatric cancer caregivers will encounter circumstances that will challenge their personal resources to face the diverse demands of care. Therefore, the objective of the study was to determine the relationship between burden syndrome and coping strategies in caregivers of pediatric cancer patients. Method: 93 main caregivers of pediatric cancer patients participated $(69.9 \%$ women, $30.1 \%$ men, mean age $=37.20$ years, $\mathrm{SD}=11.32$ ), selected in a non-probabilistic way. The Caregiver Burden Scale and the COPE-28 Questionnaire were applied. Correlational and comparative statistics were used for data analysis. Results: The results indicate the presence of significant and inverse correlations that vary between $r=-.25$ and $r=-.56$. However, no significant association was found between the dimensions of caregiver burden and substance use. On the other hand, those caregivers who

1 Paola Daniela Reyna-García. Facultad de Psicología, Universidad Peruana de Ciencias Aplicadas, Lima, Perú.

E-mail: paodrgarcia@gmail.com

2 Facultad de Ciencias de la Salud, Universidad Privada del Norte, Lima, Perú.

E-mail: tomas.caycho@upn.pe

3 Claudio Rojas-Jara. Departamento de Psicología, Universidad Católica del Maule, Talca, Chile.

E-mail: crojasj@ucm.cl

* Dirección de correspondencia: Tomás Caycho-Rodríguez. Facultad de Ciencias de la Salud, Universidad Privada del Norte Av. Alfredo Mendiola 6062, Los Olivos, Lima, Perú. E-mail: tomas.caycho@upn.pe
} 
use poorly adaptive strategies are more likely to generate overload in relation to their role, influencing their personal, family and social life. Conclusion: There is a significant relationship between overload syndrome and coping strategies. In addition, significant differences in overload are identified according to the place of origin and the hours dedicated to care.

Keywords: Caregiver overload, coping strategies, principal caregivers, pediatric cancer patients.

Sumario: 1. Introducción 2. Método 3. Resultados 4. Discusión 6. Conclusión

Como citar: Reyna-GarcíaPD, Caycho-RodríguezT, Rojas-Jara C. Síndrome de sobrecarga y estrategias de afrontamiento en cuidadores principales de pacientes oncológicos pediátricos. Psicooncología 2021; 18: 245-259, https://dx.doi.org/10.5209/psic. 77753

\section{Introducción}

A nivel mundial, el cáncer es un problema grave de salud pública ${ }^{(1,2)}$. Según la Organización Mundial de la Salud, anualmente se registra un aproximado de 400.000 casos de niños y adolescentes con cáncer ${ }^{(3)}$. En el contexto latinoamericano, se registran 17.500 nuevos casos anuales y más de 8.000 muertes derivadas de esta enfermedad ${ }^{(4)}$. En el Perú, se ha reportado un promedio de 1200 nuevos casos de cáncer infantil anualmente ${ }^{(5)}$. Por este motivo, el cáncer infantil es considerado un problema social prioritario, que no solo genera un impacto para el niño, sino también al contexto en el cual se desenvuelve ${ }^{(6)}$. Ante el diagnóstico, se genera la reestructuración familiar para identificar a la persona que proporcionará el cuidado, la asistencia, el sostenimiento y protección en el proceso de enfermedad ${ }^{(7,8)}$.

A lo largo de la enfermedad, el cuidador se encontrará con circunstancias que llegarán a desafiar sus recursos personales para afrontar las diversas demandas propias del cuidado $^{(9,10)}$. Como consecuencia, en muchas ocasiones el cuidador priorizará la salud del niño/a, comprometiendo la propia salud física y emocional ${ }^{(11)}$. Esta condición, desencadena el síndrome de sobrecarga, definido como un conjunto de reacciones y actitudes emocionales que surgen como consecuencia del cuidado a una persona dependiente ${ }^{(12)}$. En este sentido, la carga es la valoración o interpretación que mantiene el cuidador ante su rol y los estresores derivados de este, afectando directamente a la calidad de atención que le brinda al niño/a ${ }^{(13)}$. Dentro de las causas que desencadenan este síndrome se encuentran la transferencia afectiva de los problemas del enfermo hacia estos cuidadores, la repetición de aquellas situaciones conflictivas y la sensación abrumadora por la situación de cuidado ${ }^{(13)}$.

En diferentes estudios ${ }^{(14,15)}$, se han identificado variables, como la edad, el sexo, el apoyo familiar y social, la continuidad del rol, la motivación ante el rol, el vínculo con el enfermo, el grado de escolaridad del cuidador y la capacitación ante el cuidado que el niño/a necesita, que influyen en el aumento de la sobrecarga del cuidador. Esto genera que la sobrecarga sea una variable multidimensional ${ }^{(12)}$.

Ante la presencia de sobrecarga, los cuidadores utilizan diferentes estrategias de afrontamiento como medida estabilizadora ${ }^{(16)}$. Diversos autores ${ }^{(17,18)}$ consideran al afrontamiento como una forma de poder sobrellevar y adaptarse a la situación estresante. Asimismo, es planteado como un proceso dinámico entre los recursos personales y la evaluación que realice de la situación, determinando una respuesta emocional ante ésta ${ }^{(18)}$. De forma práctica, para manejar, reducir y tolerar la 
situación estresante y los sentimientos que ésta genera, se emplean las estrategias de afrontamiento ${ }^{(19)}$ que son aquellos esfuerzos cognitivos y conductuales que realizan las personas para reducir los efectos del evento estresante ${ }^{(18)}$. Carver et al. ${ }^{(20)}$ plantean 14 estrategias de afrontamiento. En primer lugar, se encuentra el afrontamiento activo, en el cual, la persona ejecuta acciones concretas para resolver el problema. La planificación, se refiere a la formulación de un plan, con pasos a seguir, para controlar la situación. Con el apoyo emocional, la persona busca la comprensión y sostenimiento del entorno. Con el apoyo social, por otro lado, la persona busca una fuente de información y consejos pertinentes sobre la situación. Religión, se refiere a la tendencia de recurrir a la religión y participar en actividades de carácter espiritual. La reinterpretación positiva brinda una mirada favorable de la situación estresante, para considerarlo como una oportunidad de crecimiento. La aceptación, refiere a la progresiva tolerancia de la situación en sí misma. La negación, por el contrario, se centra en rechazar la realidad. El humor, utiliza las bromas o la risa frente la situación estresante. La autodistracción, busca que la persona se concentre en actividades ajenas a la situación estresante. La autoinculpación, refiere a que la persona se critica y se culpa sobre el evento. La desconexión, busca reducir los esfuerzos para afrontar la situación, incluyendo la renuncia hacia las metas que están expuestas al estresor. Desahogo, refiere a la conciencia de expresar el malestar emocional ocasionado por el evento, acompañado por la acción. Por último, el uso de sustancias, se refiere a la ingesta de drogas con la finalidad de sobrellevar o sentirse mejor frente al estresor.

Ante ello, la decisión sobre qué estrategia de afrontamiento utilizar ante la situación estresante puede generar un alto impacto en la salud ${ }^{(21)}$. En este sentido, las estrategias activas utilizadas para enfrentar la situación estresante, favorecen a la regulación emocional, la resolución de problemas, la integración social y el desarrollo personal. Sin embargo, aquellas estrategias pasivas o evitativas, que presentan un pobre afrontamiento, pueden evidenciar conductas de evasión o negación, manteniendo y aumentando los niveles de estrés ${ }^{(22)}$. En el contexto de cuidadores de pacientes oncológicos pediátricos, se identifica que estos cuidadores utilizan con mayor frecuencia estrategias activas como la búsqueda de información y apoyo social. Además, en determinadas situaciones como la recaída y la intuición de muerte, los cuidadores utilizan estrategias pasivas como la evitación para manejar el impacto de la situación y sobrellevarla ${ }^{(23)}$. Por último, el nivel de instrucción, la edad y el tiempo de cuidado de niños/as, influyen en el tipo de estrategia de afrontamiento que un cuidador utilizará( ${ }^{24)}$.

Se han desarrollado líneas de investigación que relacionan ambas variables en población de cuidadores de pacientes mayores. En una investigación realizada con una población de cuidadores de personas con demencia se reconoció que existe relación entre el afrontamiento y la sobrecarga. Asimismo, se obtuvo que existe una relación significativa en las estrategias de afrontamiento de ejecución de acciones que resuelven el problema y la descarga emocional para desarrollar un menor nivel de sobrecarga ${ }^{(16)}$.

En esta misma población se identificó que utilizar estrategias de afrontamiento activo, planificación y aceptación permite que el cuidador tenga un mejor control de las demandas tanto físicas, psicológicas y socioeconómicas y, por tanto, menor nivel de sobrecarga. En contraste, utilizar estrategias más pasivas, pueden desencadenar mayores niveles de estrés y sobrecarga ${ }^{(9)}$.

Asimismo, en cuidadores de pacientes pediátricos, se reportó una relación significativa entre la sobrecarga y el afrontamiento en niños con trastorno del espectro 
autista (TEA) ${ }^{(25)}$. Sin embargo, no se ha desarrollado una línea de investigación de estas dos variables en cuidadores de niños/as con cáncer. Por lo mencionado anteriormente, este estudio cobra relevancia a nivel teórico ya que permitirá una mejor comprensión de estas dos variables en el contexto peruano y en cuidadores de pacientes oncológicos pediátricos. Esto se debe, a que, en las últimas décadas, las líneas de investigación se han encontrado dirigidas al efecto de la enfermedad oncológica en niños/as, mas no en sus cuidadores ${ }^{(7)}$. Asimismo, a nivel práctico la información servirá como fundamento para desarrollar programas de intervención preventivos para los cuidadores, debido a que presentan comportamientos desadaptativos frente a situaciones de estrés y pueden ser propensos a desarrollar depresión, agotamiento y deterioro en su calidad de vida ${ }^{(9)}$.

A partir de ello, el presente trabajo tiene como objetivo determinar la relación entre el síndrome de sobrecarga y las estrategias de afrontamiento en cuidadores de pacientes oncológicos pediátricos. Se pretende, además, comparar la variable sobrecarga en función al lugar de procedencia del cuidador y las horas dedicadas al cuidado. Mientras que las estrategias de afrontamiento se compararán entre los diferentes lugares de procedencia del cuidador.

\section{Método}

\section{Participantes}

Los participantes fueron 93 cuidadores de pacientes oncológicos pediátricos, de los cuales el $69,9 \%$ eran mujeres y el $30,1 \%$ varones, con un rango de edad entre 18 a 60 años $(M=37,20, D E=11,32)$. El $62,4 \%$ de cuidadores que se encontraban ejerciendo el rol eran madres, el $28 \%$ eran padres, el 5,4\% otro familiar y el $4,3 \%$ eran hermanos(as). En cuanto al estado civil de los cuidadores, el 47,3\% eran casados, el $25,8 \%$ solteros, el $19,4 \%$ convivientes, el $4,3 \%$ viudos y el $3,2 \%$ divorciados. Por otro lado, el $43 \%$ de los cuidadores provenía de Lima y el $57 \%$ de provincia, específicamente de departamentos como Loreto, Cusco, La libertad, Madre de Dios, entre otros. Estos cuidadores, al momento del desarrollo del estudio se encontraban en Lima para el tratamiento del menor. El 38,7\% tuvieron educación superioruniversitaria, $34,4 \%$ educación secundaria, el $14 \%$ educación superior-técnico y el $12,9 \%$ educación primaria. Con respecto a las horas dedicadas al rol, se obtuvo que el $53,8 \%$ dedicó las 24 horas al cuidado, el $22,6 \%$ de 13 a 23 horas, el $22,6 \%$ de 7 a 12 horas y el 1,1\% dedicó menos de 6 horas. Para el cuidado, el 68,8\% recibió apoyo de otros miembros de la familia y el $31,2 \%$ ha recibido apoyo de una institución (ONG, Albergue, etc.). Por último, el 76,3\% refiere que recibió alguna preparación con respecto a los cuidados que le deben brindar al paciente y el $23,7 \%$ refirió que no. Los cuidadores pediátricos oncológicos se seleccionaron a través de un tipo de muestreo no probabilístico de tipo intencional( ${ }^{(26)}$.

Finalmente, el 46,2\% de los pacientes oncológicos pediátricos eran hombres y el $53,8 \%$ mujeres, con un rango de edad entre 6 a 192 meses $(M=57,11, D E=46,61)$. Con respecto al tipo de cáncer en estos pacientes, la mayoría presentaron Leucemia Linfática Aguda (43\%), Linfoma (18,3\%) y Tumor de Wilms (12,9\%). De acuerdo con el rango del tiempo, el 39,8\% presentó la enfermedad entre 6 y 12 meses, el $38,7 \%$ entre 13 y 24 meses, el $10,8 \% 25$ y 36 meses y el $10,8 \%$ más de 37 meses. 
Por un lado, el $35,5 \%$ de los pacientes ha recibido alguna intervención quirúrgica, de los cuales $18,3 \%$ ha recibo una intervención, el $10,8 \% 2$ veces, el $5,4 \% 3$ veces y el $1,1 \% 6$ veces. Por otro lado, el $64,5 \%$ de los pacientes no ha recibido ninguna intervención quirúrgica. El 25,8\% ha sido hospitalizado en dos ocasiones, el 21,5\% 3 ocasiones, el 20,4\% en una ocasión, el 15,1\% 4 ocasiones, el 8,6\% 5 ocasiones; mientras que, el 8,6\% nunca ha pasado por el proceso de hospitalización.

\section{Instrumentos}

Ficha sociodemográfica. Esta ficha está dividida en tres áreas de información en donde se encuentran los datos personales del cuidador (sexo, edad, estado civil, lugar de procedencia y grado de instrucción), desempeño del rol de cuidador (parentesco con el paciente, horas que dedica al cuidado, si recibe apoyo familiar, de amigos o de alguna institución para el cuidado y preparación para el rol) y los datos del paciente al que le brinda los cuidados (sexo del menor, edad, tipo de cáncer, tiempo de enfermedad, intervención quirúrgica y número de hospitalizaciones).

Escala de Sobrecarga del Cuidador de Zarit et al ${ }^{(12)}$. Se utilizó la adaptación al idioma español de Martín et al. ${ }^{(27)}$. Cuenta con 22 ítems con un formato de respuesta tipo Likert con 5 opciones ( $1=$ "Nunca", 2 = "Rara vez", $3=$ "A veces", $4=$ "Frecuentemente" y 5 = "Casi siempre"). El puntaje total del instrumento varía en un rango de 22 a 110 puntos, divididos en 3 niveles: no sobrecarga ( $<47$ puntos), carga leve (47-55 puntos) y carga severa (56 puntos-110)(28). La escala está dividida en tres dimensiones: 1) Sobrecarga, que hace mención del impacto subjetivo del cuidado; 2) Competencia, que se enfoca en la percepción del cuidador sobre su capacidad para el cuidado del paciente; y 3) Dependencia, que es la percepción del cuidador sobre la dependencia de parte del paciente hacia este ${ }^{(29)}$. En este estudio, se obtuvieron índices de confiabilidad adecuados, tanto para la escala total (alfa de Cronbach $=$ ,92) y las dimensiones de sobrecarga (alfa de Cronbach $=, 90$ ), competencia (alfa de Cronbach $=, 73$ ) y dependencia (alfa de Cronbach $=, 82$ ).

Cuestionario COPE-28 de Carver et al. ${ }^{(20)}$. Se utilizó la versión adaptada al español de Morán et al. ${ }^{(18)}$ de 28 ítems, divididos en 14 dimensiones, de 2 ítems cada una. Cuenta con un formato de respuesta tipo Likert con 4 opciones, de 0 a 3 , donde $0=$ "no, en absoluto", 1 = "Un poco", 2 = "Bastante" y 3 = "Mucho". Para el presente estudio, se utilizó la versión en español de Morán, et al. ${ }^{(18)}$, y se analizarán los resultados de acuerdo con las 14 estrategias de afrontamiento propuestas inicialmente por Carver ${ }^{(20)}$. Los índices de confiabilidad de las 14 estrategias fueron adecuadas: Afrontamiento activo (alfa de Cronbach $=, 86$ ), Planificación (alfa de Cronbach $=, 84$ ), Apoyo emocional (alfa de Cronbach $=, 72$ ), Apoyo social (alfa de Cronbach $=, 91$ ), Religión (alfa de Cronbach $=, 81$ ), Reevaluación positiva (alfa de Cronbach $=, 86$ ), Aceptación (alfa de Cronbach $=, 95)$, Negación (alfa de Cronbach $=, 78)$, Humor (alfa de Cronbach $=, 90)$, Autodistracción (alfa de Cronbach $=, 88$ ), Autoinculpación (alfa de Cronbach $=, 90)$, Desconexión (alfa de Cronbach $=, 96$ ), Desahogo (alfa de Cronbach $=, 76)$ y Uso de sustancias (alfa de Cronbach $=, 75)$.

\section{Procedimiento}

En primer lugar, se revisaron los ítems de cada escala y, debido a que el COPE-28 y la escala de Zarit ya estaban validadas al español y utilizadas en el contexto peruano, se realizó una lectura de éstas. Se determinó que las preguntas eran comprensibles 
por lo que se decidió no realizar un análisis de contenido. En segundo lugar, se analizó la idoneidad de las pruebas, a través de un grupo piloto de 20 cuidadores. Para el acceso a esta población se envió la carta de presentación a la institución en donde se realizó la prueba.

Para participar del estudio, los participantes aceptaron y firmaron el consentimiento informado, especificando su participación voluntaria, el objetivo de la investigación y la confidencialidad. Posteriormente, se les brindó la ficha sociodemográfica y ambos cuestionarios. Tanto los participantes del grupo piloto como para la muestra final, realizaron los cuestionarios de manera individual, en un ambiente que se encontraba preparado para la aplicación, en un tiempo aproximado de 30 minutos.

La muestra estuvo conformada, por un lado, por cuidadores de provincia que se encontraban en un albergue. Para ello, se realizó la coordinación con la representante de la institución para la aplicación de los cuestionarios. Por otro lado, participaron cuidadores Limeños, los cuales fueron seleccionados a través de la técnica de la bola de nieve.

Finalmente, se utilizó el programa IBM SPSS Statistics 25 para realizar el análisis de los datos. En primer lugar, se calculó los estadísticos descriptivos como la media, desviación estándar, asimetría curtosis, puntaje mínimo y máximo. En segundo lugar, para establecer la correlación entre las variables se utilizó el coeficiente de correlación de Pearson. Asimismo, para comparar las variables entre dos grupos se utilizó la t de Student mientras que para comparar las mismas variables entre más de dos grupos se utilizó el coeficiente de ANOVA.

\section{Resultados}

La tabla 1 permite observar los datos descriptivos para el síndrome de sobrecarga y las estrategias de afrontamiento. Se aprecia que sobrecarga tiene una media de 44,92, competencia una media de 14,95 y dependencia tiene una media de 12. El síndrome de sobrecarga tiene una media de 71,87 que implica un nivel de carga severa. Además, en la asimetría y la curtosis se obtuvieron resultados adecuados dentro del rango +/1.5. Asimismo, el afrontamiento activo presenta el mayor valor puntaje promedio, mientras que autodistracción mostraba el menor valor. La desviación estándar de Humor y Desconexión fueron más altas a comparación de la media, lo que indica una mayor heterogeneidad en las respuestas. Además, en la asimetría y la curtosis se obtuvieron resultados adecuados dentro del rango $+/-1,5$

Tabla 1.Estadísticos descriptivos de sobrecarga y estrategias de afrontamiento y sus dimensiones.

\begin{tabular}{lcccccc}
\hline & Media & DE & Asimetría & Curtosis & Mínimo & Máximo \\
\hline Síndrome de sobrecarga & 71,87 & 12,78 &, 32 &,- 87 & 50 & 98 \\
Sobrecarga & 44,92 & 9,48 &, 42 &,- 89 & 30 & 65 \\
Competencia & 14,95 & 2,74 &,- 42 &,- 32 & 8 & 20 \\
Dependencia & 12 & 2,18 &,- 33 & -.93 & 7 & 15 \\
\hline
\end{tabular}




\begin{tabular}{lcccccc}
\hline & Media & DE & Asimetría & Curtosis & Mínimo & Máximo \\
\hline $\begin{array}{l}\text { Estrategias de } \\
\text { afrontamiento } \\
\quad \text { Afrontamiento }\end{array}$ & & & & & & \\
$\quad$ activo & 4,53 & 1,45 &,- 55 & $-1,04$ & 2 & 6 \\
$\quad$ Planificación & 4,23 & 1,71 &,- 48 & $-1,18$ & 1 & 6 \\
Apoyo emocional & 4,37 & 1,33 &,- 59 &,- 36 & 1 & 6 \\
Apoyo social & 4,31 & 1,43 &,- 23 & $-1,15$ & 2 & 6 \\
Religión & 4,42 & 1,60 &,- 57 &,- 90 & 0 & 6 \\
Reevaluación & 4,17 & 1,65 &,- 61 &,- 53 & 0 & 6 \\
positiva & 3,91 & 1,70 &,- 38 &,- 68 & 0 & 6 \\
Aceptación & 2,33 & 2,01 &, 50 & $-1,05$ & 0 & 6 \\
Negación & 1,40 & 1,95 & 1,25 &, 25 & 0 & 6 \\
Humor & 4,14 & 1,63 &,- 58 &,- 40 & 0 & 6 \\
Autodistracción & 2,86 & 1,91 &, 26 & -1 & 0 & 6 \\
Autoinculpación & 1,88 & 2,11 &, 75 &,- 81 & 0 & 6 \\
Desconexión & 2,87 & 1,92 &, 17 & $-1,02$ & 0 & 6 \\
Desahogo & 2,95 & 1,06 &, 17 & 1,16 & 0 & 6 \\
Uso de sustancias & & & & & \\
\hline
\end{tabular}

Dentro de los resultados de los análisis de correlación entre las dimensiones del síndrome de sobrecarga y afrontamiento se observa que tanto sobrecarga, dependencia y competencia, se relacionan significativamente con todas las estrategias de afrontamiento, menos con el uso de sustancias (ver Tabla 2). Específicamente, sobrecarga obtuvo asociaciones significativas e inversas con las estrategias afrontamiento activo, planificación, apoyo emocional, apoyo social, reevaluación positiva, aceptación y autodistracción. Es decir, a mayor sobrecarga menor uso de estas estrategias. Por otro lado, la sobrecarga mostró asociaciones significativas y directas con las estrategias de religión, negación, humor, desconexión, autoinculpación y desahogo. Es decir, utilizar estas estrategias se relacionan con una mayor sobrecarga. Con respecto a la dimensión competencia se obtuvieron correlaciones estadísticamente significativas con todas las estrategias de afrontamiento menos con la autodistracción y el desahogo. Por otro lado, se obtuvieron asociaciones significativas y directas con las estrategias de negación, humor, desconexión, religión y autoinculpación. Es decir, al utilizar estas estrategias es mayor la competencia. Por último, la dependencia se correlaciona significativamente con todas las estrategias de afrontamiento menos con autodistracción y religión. 
Tabla 2. Correlaciones entre dimensiones del síndrome de sobrecarga y afrontamiento

\begin{tabular}{lcccccc}
\hline & \multicolumn{2}{c}{ Sobrecarga } & \multicolumn{2}{c}{ Competencia } & \multicolumn{2}{c}{ Dependencia } \\
\hline & $r$ & $p$ & $r$ & $P$ & $r$ & $p$ \\
Afrontamiento Activo &,- 50 & $<, 001$ &,- 40 & $<, 001$ &,- 33 & $<, 001$ \\
Planificación &,- 44 & $<, 001$ & -47 & $<, 001$ &,- 35 & $<, 001$ \\
Apoyo emocional &,- 52 & $<, 001$ & -44 & $<, 001$ &,- 38 & $<, 001$ \\
Apoyo social &,- 56 & $<, 001$ &,- 53 & $<, 001$ &,- 46 & $<, 001$ \\
Religión &, 21 & $<, 001$ &, 13 &, 04 &, 16 &, 17 \\
Reevaluación positiva &,- 59 & $<, 001$ &,- 48 & $<, 001$ &,- 39 & $<, 001$ \\
Aceptación &,- 38 & $<, 001$ &,- 37 & $<, 001$ &,- 35 & $<, 001$ \\
Negación &, 72 & $<, 001$ &, 50 & $<, 001$ &, 54 & $<, 001$ \\
Humor &, 38 & $<, 001$ &, 24 & $<, 001$ &, 26 & $<, 001$ \\
Autodistracción &,- 25 &, 01 &,- 16 &, 13 &,- 15 &, 15 \\
Desconexión &, 69 & $<, 001$ &, 49 & $<, 001$ &, 43 & $<, 001$ \\
Autoinculpación &, 59 & $<, 001$ &, 50 & $<, 001$ &, 51 & $<, 001$ \\
Desahogo &,- 33 & $<, 001$ &,- 18 &, 08 &,- 28 & $<, 001$ \\
Uso de sustancias &,- 08 &, 43 &,- 15 &,- 16 &,- 04 &, 67 \\
\hline
\end{tabular}

Al comparar la sobrecarga y las estrategias de afrontamiento en función al lugar de procedencia, se encontró que tanto a nivel general de sobrecarga como la dimensión sobrecarga cuentan con una diferencia estadísticamente significativa (ver Tabla 3). Es decir, los cuidadores provenientes de provincia cuentan con un mayor nivel de sobrecarga a comparación de los cuidadores provenientes de Lima. Asimismo, se evidencia una diferencia estadísticamente significativa en la utilización de estrategias. Se identifica que existe mayor predominancia de estrategias como afrontamiento activo, planificación, apoyo emocional, apoyo social y reevaluación positiva en cuidadores limeños. Por su parte, los cuidadores de provincia utilizarían con mayor predominancia estrategias como la negación, humor, autoinculpación, desconexión y uso de sustancias.

Tabla 3. Comparación por lugar de procedencia

\begin{tabular}{lcccc}
\hline & Lima & Provincia & t & Sig \\
\hline $\begin{array}{l}\text { Síndrome de sobrecarga } \\
\text { Sobrecarga }\end{array}$ & 37,18 & 50,77 & $-9,72$ &, 00 \\
Competencia & 13,20 & 16,26 & $-6,41$ &, 28 \\
Dependencia & 10,33 & 13,26 & $-8,62$ &, 36 \\
\hline
\end{tabular}




\begin{tabular}{lcccc}
\hline & Lima & Provincia & t & Sig \\
\hline Estrategias de afrontamiento & & & & \\
Afrontamiento activo & 5,15 & 4,06 & 3,87 &, 00 \\
Planificación & 5,08 & 3,58 & 4,60 &, 00 \\
Apoyo emocional & 5,08 & 3,83 & 5,05 &, 00 \\
Apoyo social & 5,15 & 3,68 & 5,69 &, 02 \\
Religión & 4,20 & 4,58 & $-1,15$ &, 12 \\
Reevaluación positiva & 4,90 & 3,62 & 3,99 &, 00 \\
Aceptación & 4,63 & 3,38 & 3,75 &, 71 \\
Negación &, 85 & 3,45 & $-7,99$ &, 00 \\
Humor &, 28 & 2,25 & $-5,57$ &, 00 \\
Autodistracción & 4,33 & 4 &, 95 &, 76 \\
Autoinculpación & 1,88 & 3,60 & $-4,80$ &, 01 \\
Desconexión &, 35 & 3,04 & $-7,85$ &, 00 \\
Desahogo & 1,75 & 3,72 & $-5,64$ &, 66 \\
Uso de sustancias & 3 & 2,91 &, 42 &, 01 \\
\hline
\end{tabular}

Nota: el grupo provincia son aquellos cuidadores que residen fuera de lima (Loreto, Cusco, La libertad, Madre de Dios, entre otros.), pero en el momento de la encuesta se encontraban en lima para el tratamiento del menor.

Por otro lado, debido a que solo se encontró un participante en el grupo de cuidadores que dedicaban menos de 7 horas al cuidado, se optó por no considerar a este dentro del análisis. Se puede observar que existe una diferencia estadísticamente significativa entre el síndrome de sobrecarga y las horas dedicadas al cuidado. Es decir, se observa un aumento en el nivel de sobrecarga en función al incremento en las horas dedicadas al cuidado del menor (ver Tabla 4).

Tabla 4. Comparación de sobrecarga y sus dimensiones con las horas dedicadas al cuidado

\begin{tabular}{lccccc}
\hline & $\mathbf{7 - 1 2}$ horas & $\mathbf{1 3 - 2 3}$ horas & $\mathbf{2 4}$ horas & F & Sig \\
\hline Síndrome de Sobrecarga & 66,38 & 68,76 & 75,28 & 4,67 &, 01 \\
$\quad$ Sobrecarga & 41,33 & 43,05 & 47,10 & 3,38 &, 39 \\
Competencia & 13,57 & 14,29 & 15,76 & 6,05 &, 00 \\
Dependencia & 11,48 & 11,43 & 12,42 & 2,31 &, 11 \\
\hline
\end{tabular}

\section{Discusión}

El objetivo de esta investigación fue determinar la relación entre el síndrome de sobrecarga y las estrategias de afrontamiento en cuidadores de pacientes oncológicos pediátricos. Los resultados evidencian que existe una relación significativa entre el 
síndrome de sobrecarga y las estrategias de afrontamiento. En donde, si el cuidador utiliza estrategias más adaptivas, el nivel de sobrecarga es menor.

Frente a esto, investigaciones previas sobre el afrontamiento en cuidadores de niños/as con cáncer, se identifica que los cuidadores del presente estudio, utilizan estrategias activas como mediadoras de la sobrecarga, predominando las estrategias que se enfocan en la realización de acciones y el cambio cognitivo que tienen sobre la enfermedad ${ }^{(23,30,31)}$.

Al utilizar estas estrategias, los cuidadores pueden sentirse preparados para afrontar la situación de enfermedad del niño/a. En este sentido, utilizar estrategias como la aceptación de la enfermedad, puede llevar a los cuidadores a que puedan establecer un plan de acción, teniendo diferentes alternativas para poder abordar la situación ${ }^{(32)}$. Este plan no se centrará en curar al paciente, sino está orientado a buscar soluciones para reducir y controlar los síntomas secundarios de la enfermedad (13). Estos cuidadores, al utilizar estas estrategias, pueden llegar a reestructurar y cambiar la percepción que tienen sobre la enfermedad, considerándola como una oportunidad de crecimiento a nivel familiar y personal ${ }^{(23,33)}$. En este sentido, a nivel familiar, tener una nueva mirada de la enfermedad va a fortalecer los lazos entre los miembros, valorar la salud y la unión entre $\operatorname{ellos}^{(33)}$. A nivel personal, el cuidador al tener otra mirada de la enfermedad, mantendría la percepción de su autoeficacia, que da cuenta de sus capacidades para resolver problemas y la motivación para cumplir efectivamente su rol. En este sentido, tendría una mirada más objetiva de la situación que están viviendo, lo cual reduciría sus niveles de sobrecarga. Como consecuencia, el cuidador puede confiar en los procedimientos médicos presentes y futuros, reduciendo la incertidumbre, ansiedad y carga ocasionada por la enfermedad ${ }^{(32,34)}$.

Adicionalmente, utilizar estrategias como realizar actividades recreativas y de autocuidado, pueden disminuir el estrés, reduciendo la posibilidad de desarrollar sobrecarga frente a su rol de cuidado ${ }^{(23)}$. Esto se debe a que, por un momento, el cuidador se permite tener un espacio donde descentralizar su atención de la enfermedad para brindarse un tiempo propio, a las actividades de su interés y compartir con otras personas de su entorno. En este sentido, contará con mayor energía y descarga emocional y física para poder atender al niño/a.

Siguiendo la línea de las estrategias activas, diferentes autores mencionan que es importante contar con un sistema de apoyo que contenga emocionalmente al cuidador y cuente con un espacio para expresar lo que siente. Esta red de apoyo es conformada por familiares, amigos o personal médico del niño/a ${ }^{(13,35)}$. Los cuidadores, buscarán en este entorno, ser comprendidos, escuchados y sostenidos, con respecto a la situación de enfermedad. Esta proximidad con los demás, va a permitir que se sientan queridos, valorados y protegidos emocionalmente, así como sentirse admirados y respetados ${ }^{(33)}$. En este sentido, al contar con un sistema que les apoye, se disminuirían sentimientos de soledad y carga emocional; como consecuencia, se reduciría la sobrecarga emocional frente al cuidado ${ }^{(30,36)}$.

Por ende, al utilizar estrategias activas, los cuidadores pueden percibirse como más competentes ante su rol y sobreponerse ante alguna eventualidad. A diferencia, cuando los cuidadores no expresan o comunican sus complicaciones o dudas sobre el cuidado y utilizan estrategias menos adaptativas, la percepción sobre su competencia ante el rol, puede alterarse ${ }^{(24)}$.

Por otro lado, se evidencia en este estudio que el síndrome de sobrecarga puede ser desencadenado por la utilización de estrategias menos adaptativas. En este sentido, 
existen cuidadores que, ante el evento estresante, tienen dificultades para comprender el problema y reaccionar de manera adecuada. Por ello, pueden utilizar estrategias como la negación, en donde el cuidador puede ignorar o rechazar el diagnóstico del niño/a ${ }^{(32,37)}$. De igual forma, como consecuencia del diagnóstico, los cuidadores pueden experimentar sentimientos de culpa, cólera o ira frente al diagnóstico ${ }^{(38)}$. Por tal motivo, pueden criticarse y culparse de la enfermedad, afectando su desempeño frente al rol. Todos estos sentimientos, llevarían a generar estrés y desencadenar el síndrome de sobrecarga. En este sentido, la calidad de vida del cuidador se encuentra comprometida ${ }^{(39)}$. Por un lado, en su salud física puede presentar dolores musculares, alteraciones del sueño o insomnio, cansancio crónico, disminución en la respuesta inmunológica; y su salud psicológica puede verse afectada por la ansiedad, depresión y desesperanza. Por otro lado, también se perjudica la calidad en la atención brindada al niño/a ${ }^{(34)}$. Con respecto a la vida familiar, pueden surgir problemas de pareja, con los hijos y con otros miembros de la familia ${ }^{(33)}$.

En algunas investigaciones ${ }^{(32,33)}$ se evidenció que, ante la situación de enfermedad, la religión puede tener un papel fundamental en la vida de los cuidadores, tanto en el equilibrio emocional, la aceptación de la enfermedad y su tratamiento. Asimismo, esta estrategia, puede ayudar cuando el cuidador percibe que sus recursos internos (e.g., la autoeficacia y la motivación) se están agotando y se manifiesta con mayor intensidad el temor hacia la muerte del paciente ${ }^{(35)}$. Sin embargo, a pesar de que la religiosidad se puede presentar como una manera para disminuir los sentimientos de sobrecarga frente a la situación de enfermedad a través de la plegaria o la meditación, cuando los cuidadores se encuentran frente a momentos de crisis, pueden mostrarse incrédulos y con poca fe sobre la situación que están atravesando ${ }^{(33)}$, haciendo que la sobrecarga aumente en estos momentos. Esto último ha sido corroborado por el presente estudio, en el que se identificó que cuando los cuidadores utilizan la estrategia de religión se relaciona a un mayor índice de sobrecarga.

Por otro lado, se identifica al cuidador como un modelo referente para el niño/a al momento de afrontar su enfermedad ${ }^{(40)}$. Esto se debe a que, los pacientes pediátricos no logran a comprender por completo la enfermedad y buscan en estos cuidadores, referencias de estrategias a utilizar ${ }^{(7)}$. Por tal motivo, es importante que los cuidadores tengan como recurso estas estrategias adaptativas para hacer frente a la enfermedad.

Otro aspecto importante de esta investigación es que se ha identificado que las estrategias más adaptativas, son empleadas con mayor frecuencia por aquellos cuidadores que residen en Lima. En este sentido, estos cuidadores tendrían mayor apertura a estas estrategias ya que se encuentran en su lugar de origen, rodeados de familiares y amigos, los cuales los acompañan en este proceso. Asimismo, podrían contar con mayores recursos económicos para afrontar la enfermedad, reduciendo sus niveles de incertidumbre sobre el gasto de las hospitalizaciones, los procedimientos, las medicinas, las atenciones y los utensilios para el cuidado diario.

En contraste, debido a la centralización de los recursos médicos para el tratamiento del cáncer en Lima, el cuidador principal que vive en provincia, debe alejarse de su núcleo familiar y salir de su lugar de residencia para viajar y buscar alternativas de intervención y tratamiento. En este nuevo contexto de enfermedad, el cuidador se encuentra expuesto y vulnerable en un lugar desconocido y a un tratamiento complejo ${ }^{(39)}$. Asimismo, estos cuidadores no cuentan con los recursos económicos para solventar el tratamiento del niño/a. Garzón et al. ${ }^{(41)}$ señala que un bajo nivel socioeconómico es considerado como un factor negativo que influye 
en el aumento de la sobrecarga. En este sentido, el cuidador podría experimentar ansiedad frente a los gastos requeridos por la enfermedad, lo que puede influir en la calidad del cuidado.

Asimismo, estos cuidadores que vienen de provincia, se encontraban residiendo en un albergue al momento de la aplicación. A pesar de que pueden compartir sus experiencias o temores con otros cuidadores o con el personal médico de la institución, pueden sentirse solos, ya que se encuentran alejados de sus familias. Como consecuencia de este limitado sistema de apoyo pueden experimentar niveles altos en el síndrome de sobrecarga ${ }^{(42)}$.

Como dato sociodemográfico, se encontró que los cuidadores que viven el Lima, cuentan con un nivel mayor de instrucción que los cuidadores que provienen de provincia. Los cuidadores con niveles altos de instrucción, pueden comprender y llevar de mejor manera la enfermedad del niño/( ${ }^{(43)}$, así como tener estrategias activas para buscar información sobre el cuadro clínico y procedimientos; por ende, podrían presentar menores niveles de sobrecarga. Por su parte, Otavalo et al. ${ }^{(42)}$ identifican que los cuidadores que cuentan con niveles bajos de instrucción pueden tener mayores niveles de sobrecarga.

Por último, la etapa del desarrollo en la que se encuentran los pacientes oncológicos pediátricos, influye para que los cuidadores que los acompañen, brinden atención y cuidados la mayor parte del día. Una enfermedad como el cáncer, va a requerir un cuidado más riguroso y estricto, volviéndose un rol más estresante para el cuidador. Como mencionan en algunas investigaciones ${ }^{(13,44)}$, las horas dedicadas al cuidado pueden influir significativamente en el cuidado personal, salud y en la organización del tiempo. Como se puede evidenciar en este estudio, mientras más horas el cuidador le dedique al cuidado del niño/a, dedicará menos tiempo para sí mismo, para realizar otras actividades y compartir con otras personas. Estos resultados coinciden con el hallazgo de Hernández et al. ${ }^{(45)}$ en donde la cantidad de horas establecidas para el cuidado puede incrementar el riesgo de vivenciar una sobrecarga.

\section{Conclusiones}

Los resultados obtenidos plantean la relación del estrés y las estrategias empleadas por los cuidadores como un punto básico para evaluar la adaptación de este cuidador a la situación de enfermedad. Por un lado, se obtuvo que aquellos cuidadores que utilizan estrategias poco adaptativas, son más propensos a generar sobrecarga frente a su rol, influyendo en su vida personal, familiar y social. En comparación, aquellos cuidadores que utilizan estrategias más adaptativas, van a mantener su bienestar psicológico y permitirá una buena adaptación a las diferentes situaciones de la enfermedad.

La información obtenida en esta investigación, puede brindar un panorama más amplio a los trabajadores del sistema de salud sobre la vivencia y el proceso de estos cuidadores, para establecer pautas de abordaje efectivas para reducir sus niveles de estrés y ansiedad ${ }^{(42)}$. En este sentido, se pone en evidencia la relevancia de un trabajo interdisciplinario, en donde se favorezca al paciente y su familia, a nivel físico, emocional y social para mantener la calidad de vida y bienestar psicológicos de $\operatorname{estos}^{(10,41)}$.

Por último, dentro de las limitaciones del estudio, se presentó un difícil acceso a la población, ya que se requiere la aprobación de centros, albergues y hospitales 
para llegar a esta. Asimismo, no se contó con un grupo homogéneo de cuidadores de ambos sexos, por lo que sería oportuno en futuros estudios contar con muestras homogéneas de cuidadores para establecer estudios comparativos.

\section{Referencias bibliográficas}

1. Instituto Nacional del Cáncer - NIH. Estadísticas del cáncer. En línea [Acceso el 29 de mayo del 2020]. Disponible en: https://www.cancer.gov/espanol/cancer/naturaleza/ estadisticas

2. Sarria G, Limache A. Control del cáncer en el Perú: un abordaje integral para un programa de salud pública. Rev Peru Med Exp Salud Publica 2013; 30: 93-8. https:/doi. org $/ 10.17843 /$ rpmesp.2013.301.164

3. Organización Mundial de la Salud - OMS. El Cáncer infantil. En línea [Acceso el 18 de septiembre del 2019]. https://www.who.int/es/news-room/fact-sheets/detail/cancer-inchildren

4. Organización Panamericana de la Salud - OPS. El cáncer en la región de las Américas. En línea [Acceso el 15 de septiembre del 2019]. Disponible en: https:/www.paho.org/hq/ dmdocuments/2014/OPS-Nota-Informativa-Cancer-2014.pdf

5. Instituto Nacional de Enfermedades Neoplásicas - INEN. Neoplásicas recibe alrededor de 700 nuevos casos de cáncer infantil. En línea [Acceso el 8 de febrero del 2019]. Disponible en: https://portal.inen.sld.pe/neoplasicas-recibe-alrededor-de-700-nuevoscasos-de-cancer-infantil/

6. Pitillas C. Experiencia psicológica y apuntes para la intervención. En Álvarez I, De Montalvo F, editores. La familia ante la enfermedad. España, 2010; p. 21-38.

7. Rubira E, Munhoz M, Martínez M, Barbosa D, Goncalvez, A. Cuidando del niño con cáncer: Evaluación de la sobrecarga y calidad de vida de los cuidadores. Rev Latino-Am Enfermagem. 2011; 19: 512-22. https://doi.org/10.1590/S0104-11692011000300010

8. Astete J. Funcionalidad familiar y afrontamiento al estrés en padres de niños con leucemia [Tesis de grado]. Lima: Universidad San Ignacio de Loyola: 2018.

9. Flores G, Arcos M. Afrontamiento y sobrecarga subjetiva de cuidadores de pacientes con diagnóstico de demencia. Rev Psicol (Arequipa, Univ. Catól. San Pablo) 2014; 1: 41-48.

10. Gómez E, Kotliarenco M. Resiliencia familiar: un enfoque de investigación e intervención con familias multiproblemáticas. Rev Psico 2010; 19: 103-32. https://dx.doi. org $/ 10.5354 / 0719-0581.2010 .17112$

11. Ruiz-Robledillo N, Moya-Albiol L. El cuidado informal: una visión actual. REME 2012; $3(1): 22-30$.

12. Zarit S, Rever K, Bach-Peterson J. Relatives of the impaired elderly: correlates of feelings of burden. Gerontologist. 1980; 20: 649-54. https://doi.org/10.1093/geront $/ 20.6 .649$

13. Montero X, Jurado S, Méndez J. Variables que predicen la aparición de sobrecarga en cuidadores primarios informales de niños con cáncer. Psicooncología 2015; 12: 67-86. https://doi.org/10.5209/rev_PSIC.2015.v12.n1.48905

14. Martínez L, Robles T, Ramos B, Santiesteban F, García E, Morales M, García L. Carga percibida del cuidador primario del paciente con parálisis cerebral infantil severa del Centro de Rehabilitación Infantil Teletón. Rev Mex Med Fís y Rehab 2008; 20: 23-29.

15. Ramón-Arbués E, Martínez-Abadía B, Martín-Gómez S. Factores determinantes de la sobrecarga del cuidador. Estudio de las diferencias de género. Aten Prim 2017; 49: 30813. https://doi.org/10.1016/j.aprim.2016.07.003 
16. Tartaglini M, Ofman S, Stefani D. Sentimiento de sobrecarga y afrontamiento en cuidadores familiares principales de pacientes con demencia. Rev Argent Clín Psicol 2010; 19: 221-226.

17. Barquín R, Begoña M, Pérez G. El uso de estrategias de afrontamiento del estrés en personas con discapacidad intelectual. Interv Psicosoc 2018; 27: 89-94. https://doi. org/10.5093/pi2018a10

18. Morán C, Landero R, González M. COPE-28: un análisis psicométrico de la versión en español del Brief COPE. Univ Psychol 2010; 9: 543-52. https://doi.org/10.11144/ Javeriana.upsy9-2.capv

19. Sandín B. El estrés: un análisis basado en el papel de los factores sociales. Rev Int Psicol Clin y Salud. 2003; 3: 22-30.

20. Carver C. You want to measure coping but your protocol's too long: Consider the Brief COPE. Int J Behav Med 1997; 4:92-100. https://doi.org/10.1207/s15327558ijbm0401_6

21. Fernández E. Estrés percibido, estrategias de afrontamiento y sentido de coherencia en estudiantes de enfermería: su asociación con la salud psicológica y estabilidad emocional. [Tesis de grado]. León: Universidad de León: 2009.

22. Campos M, Iraurgui J, Páez D, Velasco C. Afrontamiento y regulación emocional de hechos estresantes. Un meta-análisis de 13 estudios. Bol Psicol 2004; 82: 25-44.

23. Espada M, Grau C. Estrategia de afrontamiento en padres de niños con cáncer. Psicooncología 2012; 9: 25-40. https://doi.org/10.5209/rev_PSIC.2012.v9.n1.39136

24. Campiña-Valderrama S, Duque P. Afrontamiento y adaptación de cuidadores de niños y niñas con cáncer. Univ Salud 2016; 18: 302-311. https://doi.org/10.22267/rus.161802.40

25. Beas L. Estrés por sobrecarga y afrontamiento en padres de niños con trastorno del espectro autista. [Tesis de grado]. Lima: Universidad Cesar Vallejo: 2018.

26. Hernández R, Fernández $\mathrm{C}$, Baptista $\mathrm{M}$, editores. Metodología de la investigación; Cuidad de México, 2010.

27. Martín, M, Otermin P, Pérez V, Pujol J, Martín M, Gobartt A, Pons S, Balaña M. EDUCA study: Psychometric properties of the Spanish version of the Zarit Caregiver Burden Scale. Aging Ment Health 2010; 14: 705-11. https://doi.org/10.1080/13607860903586094

28. Hidalgo L. Sobrecarga y afrontamiento en familiares cuidadores de personas con esquizofrenia. [Tesis de grado]. Lima: Pontificia Universidad Católica del Perú: 2013.

29. Albarracín A, Cerquera A, Pabón D. Escala de sobrecarga del cuidador Zarit: estructura factorial en cuidadores informales de Bucaramanga. Rev Psic Univ Antioquia. 2016; 8: 87-99. https://doi.org/10.17533/udea.rpsua.v8n2a06

30. Clake N, McCarthy M, Downie P, Ashley D, Anderson V. Gender differences in the psychosocial experience of parents of children with cancer: a review of the literature. Psychooncology 2009; 18: 907-15. https://doi.org/10.1002/pon.1515

31. Moral J, Martínez J. Reacción ante el diagnóstico de cáncer en un hijo: estrés y afrontamiento. Psicol Salud 2009; 19: 189-196. https://doi.org/10.25009/pys.v19i2.628

32. García B. Estudio cualitativo del perfil del cuidador primario, sus estilos de afrontamiento y el vínculo afectivo con el enfermo oncológico infantil. [Tesis doctorado]. Barcelona: Universitat Ramon Llul: 2011.

33. Oliveira A, Schwartz E, Machado B, Burille A, Vestena J, Machado A. Rede socia e vínculos apoiadores das familias de crianças com cancer. Texto Contexto Enferm 2010; 19: 334-42. https://doi.org/10.1590/S0104-07072010000200015

34. Pablo R, Domínguez B, Peláez V, Rincón S, Orea A. Sobrecarga y autoeficacia percibida en cuidadores primarios informales de pacientes con insuficiencia cardíaca. Rev Clin Med Fam 2016; 9: 152-158. 
35. Almico T, Faro A. Enfrentamiento de cuidadores de crianças com cancer em proceso de quimioterapia. Psicol Saúde Doenças. 2014; 15: 723-37. https://doi. org/10.15309/14psd150313

36. Pérez A. El afrontamiento en familias de niños con cáncer: un estado del arte. [Tesis de grado]. Barrancabermeja: Universidad Cooperativa de Colombia: 2018.

37. Nastoyashchaya E. Trastornos afectivos y evitación experiencial en padres y familiares de niños con cáncer. [Tesis de maestría]. Oviedo: Universidad de Oviedo: 2016.

38. Arca M, Cachay J. Cuidado enfermero frente al afrontamiento en padres de preescolares con leucemia de un hospital nacional [Tesis de grado]. Lambayeque: Universidad Nacional Pedro Ruiz Gallo: 2017.

39. Cristina E, Rangel L, Santos M, Pereira M. Sobrecarga y calidad de vida de madres de niños y adolescentes con enfermedad crónica: revisión integradora. Rev Latino-Am Enferm 2015; 23: 769-77. https://doi.org/10.1590/0104-1169.0196.2613

40. Alonso C. El impacto del cáncer infantil en el contexto familiar desde la experiencia vital de los/as progenitores/as. [Tesis de grado]. Santa Cruz de Tenerife: Universidad de La Laguna: 2015.

41. Garzón-Maldonado FJ, Gutiérrez-Bedmar M, García-Casares N, Pérez-Errázquin F, Gallardo-Tur A, Martínez-Valle MD. Calidad de vida relacionada con la salud en cuidadores de pacientes con enfermedad de Alzheimer. Neurología 2017; 32: 508-15. https://doi.org/10.1016/j.nrl.2016.02.023

42. Otavalo M, Banegas K. Prevalencia del síndrome de sobrecarga y las estrategias de afrontamiento en los cuidadores primarios de pacientes oncológicos pediátricos en el centro de acogida de niños con cáncer "Emaus". [Tesis de grado]. Cuenca: Universidad del Azuay; 2019.

43. Escudero-Rodríguez B, Díaz-Álvarez E, Pascual-Cortés O. Cuidadores informales: necesidades y ayudas. Rev Rol Enferm 2001; 24:183-9.

44. Williams A. Family caregivers to adults with cancer. The consequences of caring. Psychooncology 2018; 210: 87-103. https://doi.org/10.1007/978-3-319-64310-6_6

45. Hernández N, Salas A, Altuve J. Afrontamiento, funcionamiento familiar y calidad de vida relacionada con la salud en cuidadores venezolanos de pacientes con cáncer. Psicol Salud 2020; 30: 161-72. https://doi.org/10.25009/pys.v30i2.2651 
